\title{
Therapeutic Response of Metastatic Colorectal Cancer Harboring a KRAS Missense Mutation After Combination Chemotherapy With the EGFR Inhibitor Panitumumab
}

\author{
Emil Lou, MD, PhD; ; Donna D'Souza, MDª ; and Andrew C. Nelson, MD, PhDc
}

\begin{abstract}
Over the past decade, subset analyses of retrospective and prospective clinical studies have determined that $K R A S$-mutated metastatic colorectal cancers do not respond effectively to inhibition of epidermal growth factor receptor (EGFR) with the EGFR-targeting monoclonal antibodies cetuximab or panitumumab. Within the past few years, the scope of tested variants in the KRAS oncogene has expanded significantly, and testing of all RAS family genes has become more widely available in clinical laboratories. Expert consensus guidelines have recommended not using EGFR inhibitors in patients with KRAS-mutated tumors. However, with increasing identification of low-prevalence variants, it is conceivable that some RAS mutations do not provide equivalent resistance to EGFR inhibition compared with the most prevalent mutations at codons 12,13 , and 61 . This report describes a case of a patient with metastatic colon cancer harboring the p.A59T variant of KRAS, with objective radiographic response (36\% decrease per RECIST 1.1) and carcinoembryonic antigen biomarker response to panitumumab therapy given with FOLFIRI chemotherapy. We propose that A59T represents one potential exception to the guidelines that KRAS mutant tumors fail to respond to therapy with EGFR inhibitors, altering the paradigm of using this generalized approach.
\end{abstract}

KRAS mutations are present in $33 \%$ to $40 \%$ of cases of colorectal cancer (CRC). ${ }^{1-3}$ Numerous KRAS mutations have been identified thus far, with the most common forms being missense mutations of glycine to aspartic acid at codons 12 (G12D) or 13 (G13D) or glycine to valine at codon $12(\mathrm{G} 12 \mathrm{~V}) .{ }^{1}$ These 3 forms have traditionally constituted $>75 \%$ of all known KRAS mutations.

Over the past decade, a number of retrospective studies have established that patients whose tumors harbor KRAS mutations do not benefit from treatment with epidermal growth factor receptor (EGFR) inhibitors. ${ }^{4}$ During this time, there have been investigations examining whether this is broadly and universally true across all forms of KRAS mutations, or whether any exceptions exist. De Roock et $\mathrm{al}^{2}$ hypothesized that

From the Departments of aedicine, Division of Hematology, Oncology,

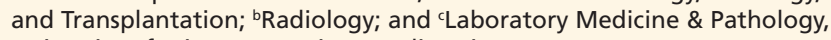
University of Minnesota, Minneapolis, Minnesota.

Submitted August 24, 2016; accepted for publication January 23, 2017.

The authors have disclosed that they have no financial interests, tumors with the G13D mutation specifically respond better to cetuximab than do other mutations, including other point mutations within the same codon. The authors performed a retrospective study of 7 clinical trials (EVEREST, BOND, SALVAGE, BABEL, CO.17, MABEL, and EMR202600). The subanalysis of the pooled population of 579 patients treated with cetuximab suggested that the 32 patients with G13D mutations had both longer progression-free survival (PFS) and overall survival (OS) compared with those with other KRAS mutations. ${ }^{2}$ However, a recent prospective phase II trial of patients with chemorefractory KRAS G13D mutation-positive CRCs treated with cetuximab with or without irinotecan (ICECREAM) showed no statistically significant difference in disease control at

arrangements, affiliations, or commercial interests with the manufacturers of any products discussed in this article or their competitors.

Correspondence: Emil Lou, MD, PhD, University of Minnesota, Division of Hematology, Oncology, and Transplantation, Mayo Mail Code 480, 420 Delaware Street SE, Minneapolis, MN 55455. E-mail: emil-lou@umn.edu 
6 months. ${ }^{5}$ This finding underscores the need for prospective evaluation of efficacy. At the same time, identification of more variants of RAS has predicated a call for extended RAS gene mutation testing for patients with metastatic CRC. ${ }^{6}$ The recommendation from the 2015 ASCO Provisional Clinical Opinion Update was to perform testing of KRAS and NRAS exons 2 (codons 12 and 13), 3 (codons 59 and 61), and 4 (codons 117 and 146), casting a wider net than in previous years. ${ }^{6}$ One can postulate that discoveries of RAS variants may yet open the door to versions that actually subvert the paradigm of lack of response of these tumors to EGFR inhibition.

This case report describes a female patient presenting with metastatic CRC whose tumor was initially thought to be wild-type for KRAS after limited testing of exon 2 only. After radiologic progression of disease per RECIST, version $1.1{ }^{7}$ and also an increase in carcinoembryonic antigen (CEA) biomarker while on combination chemotherapy (FOLFIRI with bevacizumab), bevacizumab was discontinued in favor of initiating an EGFR inhibitor (panitumumab) while continuing the FOLFIRI backbone. This alteration to her treatment approach led to a radiographic partial response $(36 \%)$ and a decline in the serum CEA biomarker, with further stable disease of her metastatic tumors over an overall period of 8 months. On initiation of all-RAS (KRAS, NRAS, and HRAS) testing at our institution, further analysis of her tumor detected a KRAS missense mutation (A59T), making her objective clinical response to EGFR inhibition all the more remarkable.

\section{Case Report}

A 50-year-old Caucasian woman with a history of asthma presented with radicular and low back pain. MRI performed of the pelvis and sacrum with and without contrast showed large infiltrative marrowreplacing lesions in the right sacrum and S2 vertebral level, partially extending into the spinal canal and encasing the exit of S2 and S3 nerve roots. CT scan of the chest characterized a $1.1 \times 1.2-\mathrm{cm}$ right middle lobe nodule, consistent with potentially a primary bronchogenic carcinoma versus metastatic colon cancer. There was also a tiny $0.3-\mathrm{cm}$ indeterminate nodular density in the left lower lung and multiple hypodense liver lesions in the liver consistent with metastasis.
A CT-guided biopsy of a liver lesion confirmed a metastatic adenocarcinoma consistent with primary colorectal malignancy. A PET/CT performed before treatment demonstrated interim growth of the right middle lobe pulmonary mass, which was hypermetabolic. There were $>10$ hypermetabolic lesions in the liver, increasing in size and number, consistent with aggressive disease. The sacral metastasis and medial right iliac metastasis at the level of the sacroiliac joint were also hypermetabolic, as were enlarged mesenteric lymph nodes. A colonoscopy confirmed invasive moderately differentiated adenocarcinoma, a near-circumferential mass at the rectosigmoid junction.

The patient's family history was notable for several cases of cancer. One sister was diagnosed with adenoid cystic salivary gland cancer at the age of 50 years. Her mother was diagnosed with ovarian cancer at age 49 years, and at approximately 65 years of age was diagnosed with melanoma that was superficial and resected. Her maternal grandfather had colon cancer in his 80 s and died of this disease shortly thereafter.

\section{Treatment Course}

The patient received a short course of palliative radiation to the sacral/iliac metastasis for pain control (3000 Gy over 10 fractions), followed by a first-line course of palliative chemotherapy with mFOLFOX-6 chemotherapy and bevacizumab. Radiologic evaluation after 4 cycles demonstrated a radiologic partial response to treatment, with decreased size of pulmonary metastases and decreased size of multiple hepatic metastases (overall 30\% decrease per RECIST criteria). She also received a bisphosphonate (zolendronic acid) due to her bone metastasis. However, after 2 further cycles on therapy, she developed rectal bleeding and bevacizumab was discontinued. A CT performed soon thereafter to evaluate new pain revealed 3 new pleural lesions, consistent with progressive metastatic disease. Second-line FOLFIRI chemotherapy was initiated for 7 cycles, with overall stable response until further progression of lung and liver metastases (growth of some existing lesions and several new hepatic and pulmonary lesions). Bevacizumab was added to her FOLFIRI regimen, and her disease burden remained stable per RECIST criteria for an additional 6 months until radiographic progression occurred, marked by growth of several 
nontarget lesions. Concurrently, the CEA biomarker had increased from $15.8 \mathrm{mcg} / \mathrm{L}$ at the time of initiation of FOLFIRI to $69.6 \mathrm{mcg} / \mathrm{L}$ (Figure 1).

Because the patient had been experiencing intermittent mild epistaxis over several months, attributed to the bevacizumab and exacerbated by 5-FU-induced mucositis, options for moving forward with treatment were discussed. For that reason, bevacizumab was discontinued and panitumumab was added to her FOLFIRI chemotherapy regimen. After 4 cycles of this new combination, CT scan demonstrated a partial response to therapy (36\% decrease per RECIST), as evidenced by decreased size and/or cavitation of the pulmonary metastases, and stable to mildly decreased size of multiple hepatic metastases (Figure 2). She continued on this regimen for a total of 8 months, with good clinical response and radiologically stable disease, before experiencing radiologic and CEA biomarker disease progression. After this progression, she initiated third-line therapy with the multikinase inhibitor regorafenib at a modified dose ( $80 \mathrm{mg} / \mathrm{d}$ orally). Unfortunately, she sustained radiographic and clinical progression of disease, and an increase in CEA, after only 2 months of treatment with this drug. As of this writing, she has initiated next-line treatment on a phase I clinical protocol that includes treatment with a histone deacetylase inhibitor and a PD-L1 inhibitor.

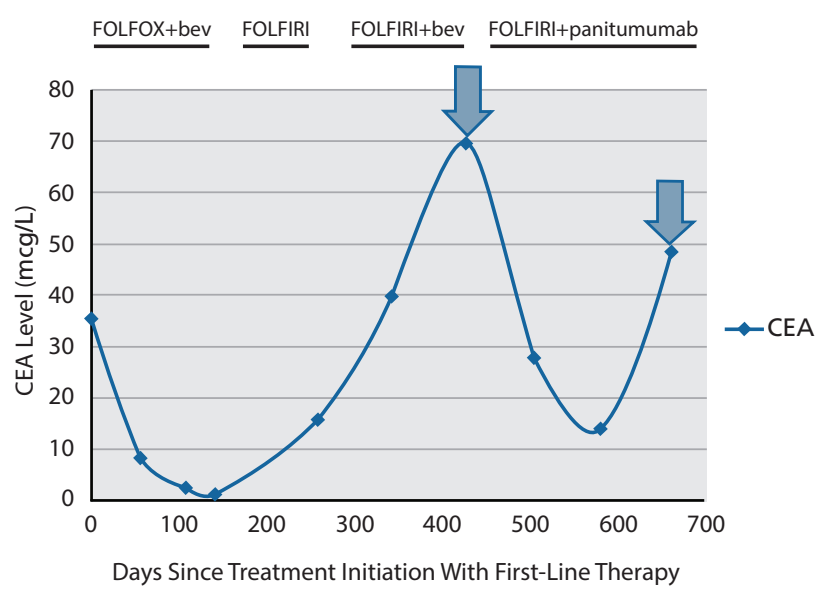

Figure 1. Fluctuation and response of serum CEA biomarker throughout her treatment course, including the 8-month period during which her cancer responded to treatment with panitumumab and FOLFIRI chemotherapy (treatment interval marked by solid blue arrows). That interval of treatment also resulted in a radiographic partial response (36\% per RECIST-based calculations).

Abbreviations: Bev, bevacizumab; CEA, carcinoembryonic antigen.

\section{Morphologic Findings}

CT-guided core needle biopsies were simultaneously obtained from one of the liver lesions and the sacral mass identified on prior imaging to provide the initial pathologic diagnosis. Both biopsies showed moderately differentiated adenocarcinoma with both intraluminal and geographic areas of necrosis, neutrophilic infiltrate, and frequent mitotic figures (Figure 3A,B). There was moderate desmoplasia surrounding the metastatic carcinoma. The elongated nuclear contours and glandular profiles were consistent with a colorectal origin. Immunohistochemical stains (Figure 3C-E) for cytokeratin 20 and CDX2 were diffusely and strongly positive in the malignant cells, whereas cytokeratin 7, estrogen receptor, and CA19-9 were negative, providing evidence for the diagnosis of metastatic CRC.

Colonoscopy confirmed a near-circumferential mass at the rectosigmoid junction, an invasive adenocarcinoma that was also moderately differentiated and morphologically consistent with the previously biopsied metastatic sites (Figure 4A). Immunohistochemistry for mismatch repair proteins showed intact expression of MLH1, PMS2, MSH2, and MSH6 (Figure 4B-E), thus indicating that the tumor likely had a microsatellite stable phenotype.

\section{Molecular Testing}

Formalin-fixed paraffin embedded tissue from the primary tumor was initially performed by Sanger sequencing of KRAS exon 2, which was the institutional standard of practice at the time for metastatic CRC. This analysis did not detect any alterations in the coding DNA sequence of codons 12 or 13 (data not shown). Several months later, the institution switched standard-of-care practice to a multiplexed, amplicon-based next-generation sequencing (NGS) panel, including exons 2 and 3 of $K_{-}, N_{-}$, and HRAS genes, exon 15 of BRAF, and exons 2, 5, 9, 10, 20, and 21 of PIK3CA. The patient's banked DNA sample was retested, and a KRAS: c.175G >A (p.Ala59Thr) mutation was identified (see supplemental eFigure 1, available with this article at JNCCN.org), which is predicted to cause a missense substitution of threonine for alanine at codon 59 in exon 3 of the KRAS protein. The p.A59T mutation was clinically reported as likely pathogenic-based on review of the available evidence. No other alterations were detected in the focused NGS CRC panel. 
Lou et al

A

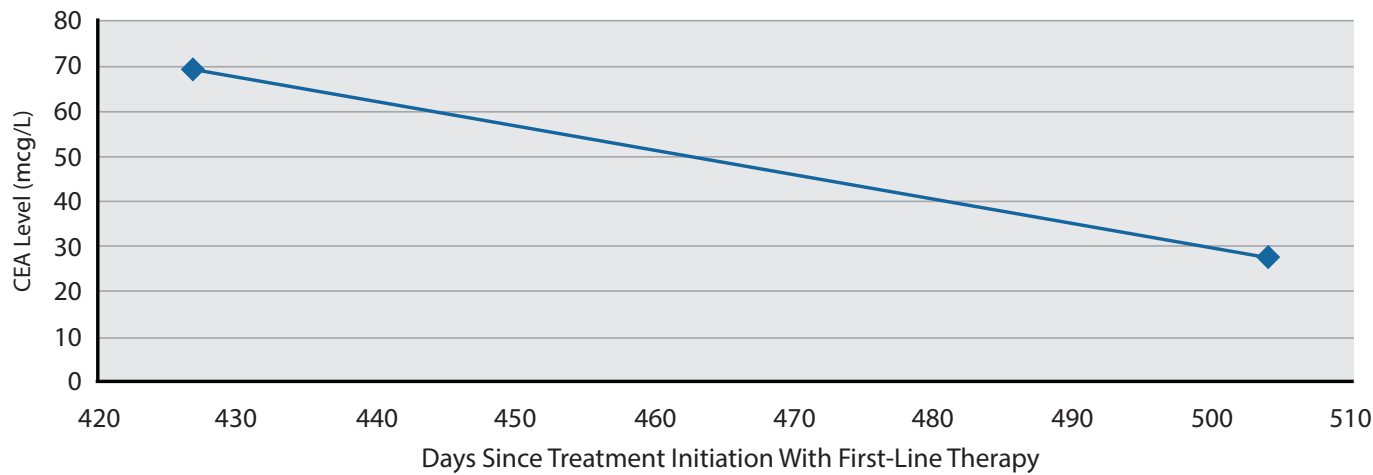

B
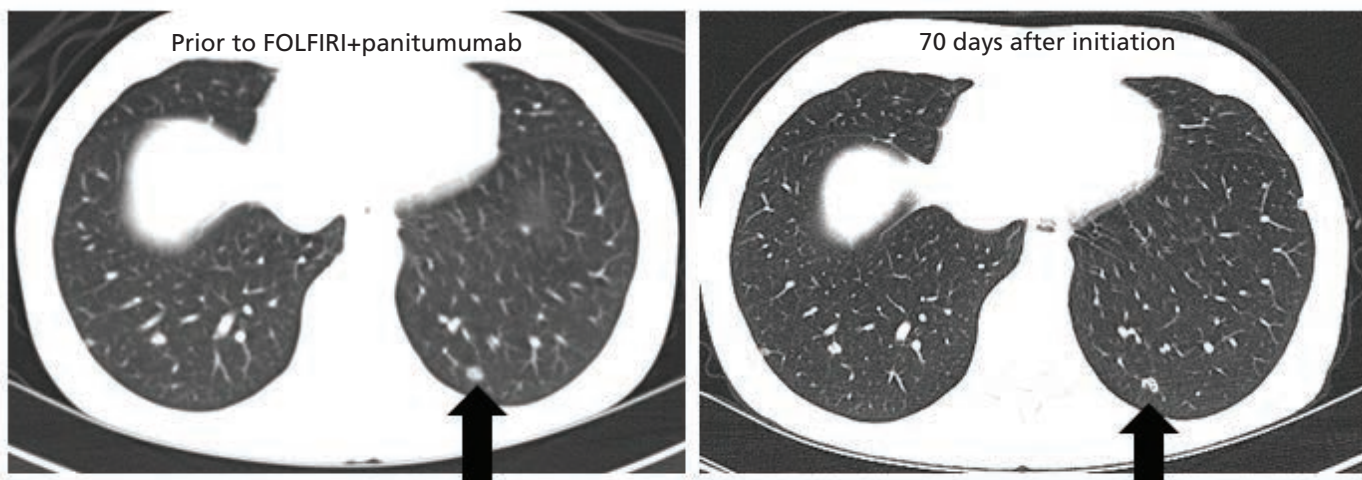

C
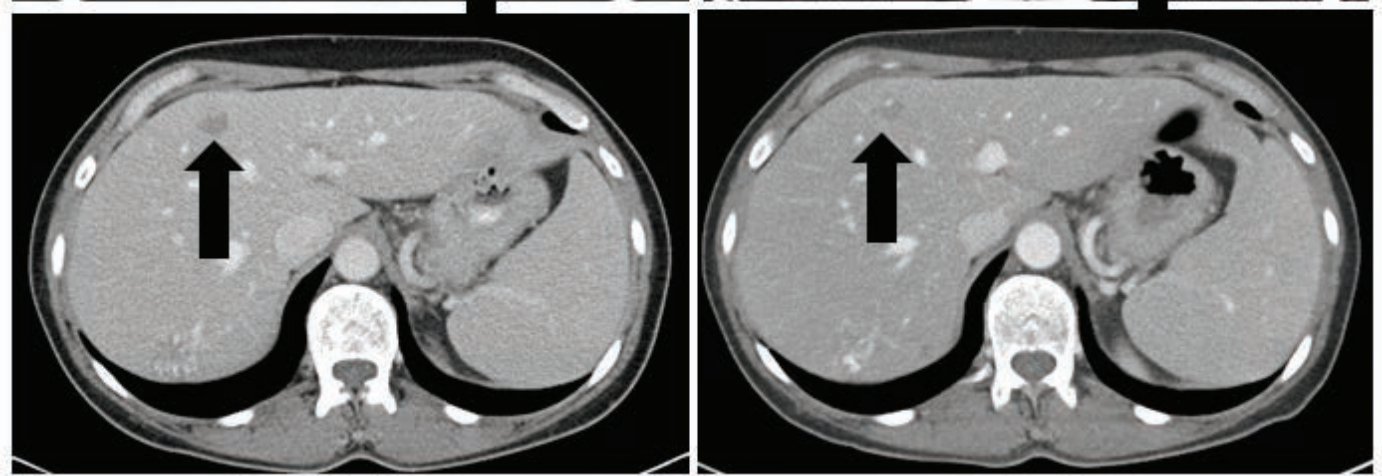

Figure 2. Representative sample of radiologic tumor responses to this patient's treatment with panitumumab in combination with FOLFIRI chemotherapy backbone. Her overall radiologic response was a $36 \%$ decrease in size of the target lesions compared with baseline measurements, making this a partial response per RECIST. (A) Serum carcinoembryonic antigen (CEA) levels corresponding to the CT scans shown, before and after treatment using panitumumab and FOLFIRI. (B) Representative right lower lobe metastatic pulmonary nodule. In this example, the nodule is similar in size but more cavitary compared with baseline. (C) Representative hepatic metastasis (right-sided/segment 7) that also responded to this combination therapy. Black arrows indicate the representative lesions before (left-hand panel) and after 4 cycles (right-hand panel) of this line of therapy.

\section{Discussion}

With the expansion of NGS and all-RAS testing within the past few years, molecular profiling of CRC tumors has played an increasingly important role in determining optimal therapy for patients.

EGFR inhibition with cetuximab has been demonstrated to benefit patients with KRAS wild-type tumors, and has entered the repertoire of standard treatment for metastatic CRC that does not harbor known pathogenic KRAS mutations. ${ }^{8}$ Further studies combining cetuximab with chemotherapy (FOLFOX-4, FOLFOX-6, or FOLFIRI) stratified patient subsets according to wild-type or pooled mutation status of KRAS, and demonstrated clear differences in benefit as assessed by response rates, PFS, and OS. ${ }^{9,10}$ Current guidelines recommend broadly that patients with KRAS mutation-positive CRC should not be treated with EGFR inhibitors, such as cetuximab or panitumumab. ${ }^{5}$ However, by pooling all KRAS mutations in the same category, are we performing the medical equivalent of throwing out 


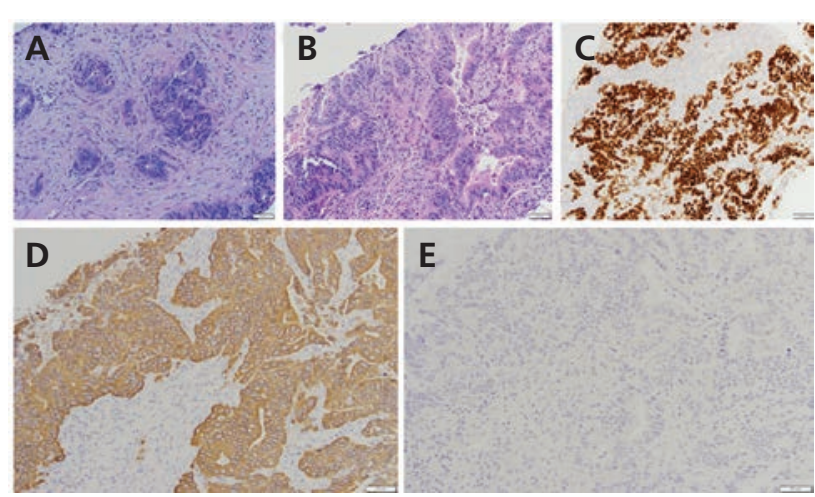

Figure 3. Histopathology evaluation of the metastatic tumors. (A) Hematoxylin-eosin (H\&E) stain of metastatic liver lesion (original magnification $\times 20$ ). (B) H\&E stain of metastatic sacral mass, with immunohistochemical stains demonstrating expression of (C) CDX-2 and (D) cytokeratin 20, with (E) absence of cytokeratin 7 (all images: original magnification $\times 20$ ). This immunophenotype is consistent with a colorectal primary.

the baby with the bath water? Or are all KRAS mutations truly equal?

Panitumumab, another humanized monoclonal antibody directed against EGFR, was FDA-approved in 2006 for the treatment of progressive metastatic CRC after failure of fluorouracil, oxaliplatin, and irinotecan-based regimens. ${ }^{11}$ This drug has come under careful examination for use in multiple clinical scenarios for CRC. Peeters et $\mathrm{a}^{12}$ presented a retrospective analysis of 3 phase III trials encompassing panitumumab as single-agent therapy or with the FOLFOX-4 or FOLFIRI regimens in first- or secondline therapy. Patient cohorts were stratified by wildtype KRAS or presence of KRAS mutations in codons 12 or 13 ( 7 alleles in total, including G13D). Results analyzing specific alleles were inconsistent with prior findings by De Roock et $\mathrm{al}^{2}$ in their analysis on cetuximab: patients with G13D displayed shorter OS when treated with panitumumab and FOLFOX-4 compared with other groups. Interestingly, patients with G12V had a favorable OS on this regimen. These data raised interesting questions about the validity of separating G13D from other mutations, and even the possible subtle underlying differences in mechanism between cetuximab and panitumumab, which might explain these different, and apparently conflicting, findings.

The p.A59T KRAS mutation has been reported in a small number of human CRC cases (COSMIC database ${ }^{13,14}$ and The Cancer Genome Atlas $\left[\right.$ TCGA] ${ }^{15,16}$ ). The alteration was deposited by one submitter in NCBI's ClinVar based on a published report identifying the mutation in a xenograft from a human transitional cell carcinoma of the bladder. ${ }^{17}$ Other missense variants affecting codon 59 have also been reported in ClinVar, COSMIC, and TCGA. Furthermore, this codon is located in the switch 2 domain of KRAS, which is critical to GTP hydrolysis, and there is no evidence of nonsilent polymorphisms in this region within the Exome Aggregation Consortium (ExAC) database ${ }^{18}$ of normal human genomic variation. Finally, the A59T mutation has not been reported in association with Noonan syndrome or cardiofaciocutaneous syndrome, which are 2 autosomal dominant inherited diseases caused in a minority of cases by KRAS germline mutations. These lines of evidence demonstrate that the A59T mutation occurs somatically in human cancers, has not been identified as a germline alteration, and is located in a region that is biochemically relevant to cancer pathogenesis.

Modeling of this alteration using the Mutation Assessor in silico algorithm ${ }^{19}$ predicted a high functional impact (FI score $=3.59$ ), and a search of PubMed identified one in vitro study ${ }^{20}$ using the SNU175 human colon cancer cell line, which carries the A59T KRAS mutation. The in vitro data suggest that the SNU175 (KRAS A59T) cell line has a higher proliferative fraction in the face of cetuximab treatment versus the KRAS wild-type human CRC cell lines DiFi and SW48, but direct statistical comparisons of these groups treated with equivalent drug concentrations were not performed. Therefore, the A59T mutation was interpreted as a

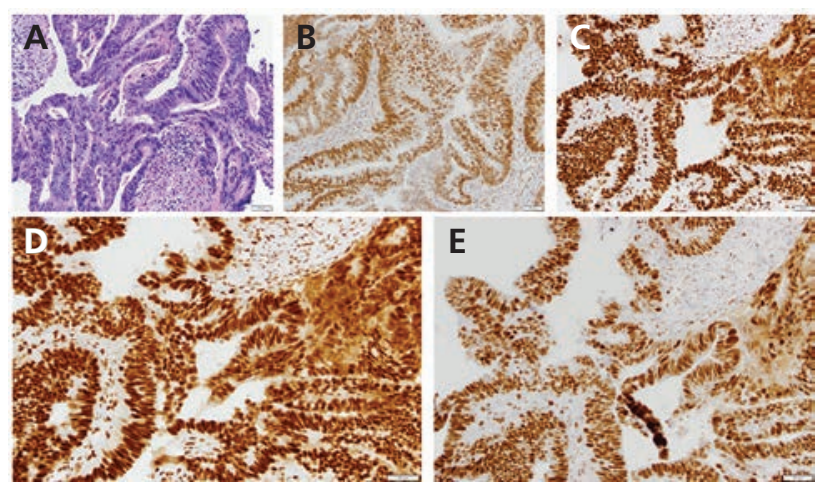

Figure 4. Histopathologic confirmation of the primary tumor and assessment for microsatellite instability. (A) Hematoxylin-eosin stain of primary colon tumor (original magnification $\times 20$ ). (B-E) Immunohistochemistry (original magnification $\times 20$ ) shows protein expression of (B) MLH1, (C) PMS2, (D) MSH2, and (E) MSH6. Expression of all 4 proteins is consistent with a microsatellite stable tumor phenotype and essentially rules out an association with Lynch syndrome. 
Lou et al

potentially pathogenic mutation, ${ }^{21}$ but the clinical utility $^{22}$ of this result to guide individual therapeutic decision-making was reported as uncertain given the paucity of clinical outcome data on patients with this specific variant. ${ }^{23}$

\section{Conclusions}

We observed a remarkable clinical, radiographic, and CEA biomarker response of a patient with metastatic CRC harboring the A59T missense mutation of KRAS treated for 8 months with panitumumab and a FOLFIRI backbone. Further investigation is warranted to determine whether this missense mutation represents a potential exception to the general rule that tumors harboring KRAS mutations are nonresponsive to EGFR inhibition.

\section{Acknowledgments}

The authors wish to thank the patient for providing written consent to publish her case.

\section{References}

1. Neumann J, Zeindl-Eberhart E, Kirchner T, Jung A. Frequency and type of KRAS mutations in routine diagnostic analysis of metastatic colorectal cancer. Pathol Res Pract 2009;205:858-862.

2. De Roock W, Jonker DJ, Di Nicolantonio F, et al. Association of KRAS p.G13D mutation with outcome in patients with chemotherapyrefractory metastatic colorectal cancer treated with cetuximab. JAMA 2010;304:1812-1820.

3. Oliveira C, Westra JL, Arango D, et al. Distinct patterns of KRAS mutations in colorectal carcinomas according to germline mismatch repair defects and hMLH1 methylation status. Hum Mol Genet 2004;13:23032311.

4. Allegra CJ, Jessup JM, Somerfield MR, et al. American Society of Clinical Oncology provisional clinical opinion: testing for KRAS gene mutations in patients with metastatic colorectal carcinoma to predict response to anti-epidermal growth factor receptor monoclonal antibody therapy. J Clin Oncol 2009;27:2091-2096

5. Segelov E, Thavaneswaran S, Waring PM, et al. Response to cetuximab with or without irinotecan in patients with refractory metastatic colorectal cancer harboring the KRAS G13D mutation: Australasian Gastro-Intestinal Trials Group ICECREAM Study. J Clin Oncol 2016;34:2258-2264.

6. Allegra CJ, Rumble RB, Hamilton SR, et al. Extended RAS gene mutation testing in metastatic colorectal carcinoma to predict response to antiepidermal growth factor receptor monoclonal antibody therapy: American Society of Clinical Oncology provisional clinical opinion update 2015. J Clin Oncol 2016;34:179-185.

7. Eisenhauer EA, Therasse P, Bogaerts J, et al. New response evaluation criteria in solid tumours: revised RECIST guideline (version 1.1). Eur J Cancer 2009;45:228-247.

8. Van Cutsem E, Kohne CH, Hitre E, et al. Cetuximab and chemotherapy as initial treatment for metastatic colorectal cancer. N Engl J Med 2009;360:1408-1417.

9. Bokemeyer C, Bondarenko I, Hartmann JT, et al. Efficacy according to biomarker status of cetuximab plus FOLFOX-4 as first-line treatment for metastatic colorectal cancer: the OPUS study. Ann Oncol 2011;22:1535_ 1546.

10. Folprecht G, Gruenberger $\mathrm{T}$, Bechstein WO, et al. Tumour response and secondary resectability of colorectal liver metastases following neoadjuvant chemotherapy with cetuximab: the CELIM randomised phase 2 trial. Lancet Oncol 2010;11:38-47.
11. Giusti RM, Cohen MH, Keegan P, Pazdur R. FDA review of a panitumumab (Vectibix) clinical trial for first-line treatment of metastatic colorectal cancer. Oncologist 2009;14:284-290.

12. Peeters M, Douillard JY, Van Cutsem E, et al. Mutant (MT) KRAS codon 12 and 13 alleles in patients (pts) with metastatic colorectal cancer (mCRC): assessment as prognostic and predictive biomarkers of response to panitumumab (pmab) [abstract]. J Clin Oncol 2012;30(Suppl 4):Abstract 383.

13. Forbes SA, Beare D, Gunasekaran P, et al. COSMIC: exploring the world's knowledge of somatic mutations in human cancer. Nucleic Acids Res 2015;43(Database issue):D805-811.

14. COSMI database. Available at: http://cancer.sanger.ac.uk/cosmic Accessed March 3, 2017.

15. Gao J, Aksoy BA, Dogrusoz U, et al. Integrative analysis of complex cancer genomics and clinical profiles using the cBioPortal. Sci Signal 2013;6:pl1.

16. The Cancer Genome Atlas. Available at: http://www.cbioportal.org/. Accessed August 1, 2016

17. Grimmond SM, Raghavan D, Russell PJ. Detection of a rare point mutation in Ki-ras of a human bladder cancer xenograft by polymerase chain reaction and direct sequencing. Urol Res 1992;20:121-126.

18. Exome Aggregation Consortium. Available at: http://exac.broadinstitute. org. Accessed August 1, 2016.

19. Reva B, Antipin Y, Sander C. Predicting the functional impact of protein mutations: application to cancer genomics. Nucleic Acids Res 2011;39:e118.

20. Lee J, Lee I, Han B, et al. Effect of simvastatin on cetuximab resistance in human colorectal cancer with KRAS mutations. J Natl Cancer Inst 2011;103:674-688.

21. Richards $S$, Aziz N, Bale $S$, et al. Standards and guidelines for the interpretation of sequence variants: a joint consensus recommendation of the American College of Medical Genetics and Genomics and the Association for Molecular Pathology. Genet Med 2015;17:405-424.

22. Joseph L, Cankovic M, Caughron S, et al. The spectrum of clinical utilities in molecular pathology testing procedures for inherited conditions and cancer: a report of the Association for Molecular Pathology. J Mol Diagn 2016;18:605-619

23. Douillard JY, Oliner KS, Siena S, et al. Panitumumab-FOLFOX4 treatment and RAS mutations in colorectal cancer. N Engl J Med 2013;369:10231034.

\section{See JNCCN.org for supplemental online content.}

